schlagen. Die Früchte reifen erst im zweiten Jahr. Ilex verbreitet sich durch Wurzelausschlag und durch Samen, die aber erst die Verdauungsorgane eines Tieres passiert haben müssen (endozoische Verbreitung). Der Same geht erst in 11/2-2 Jahren auf. Der Redner fand nie einen Hülsenkeimling im freien Gelände und vertritt die Ansicht, daß die endozoische Verbreitung bei uns wegfüllt. Eine große Ilexgärtnerei bei Remscheid bezieht zur Zucht Samen aus Italien, Frankreich und Holland. 8-14 tägige Gärung der eingestampften Samen beschleunigt die Keimung. Bei Verpflanzungen kommen nur $30-40 \%$ fort. Durch die vielseitige Verwendung, die Laub, Früchte, Rinde und Holz finden, ist die Hülse der Gefahr des Aussterbens ausgesetzt. Um sie als Naturdenkmal zu erhalten, muB sie unter Schutz gestellt werden, wie es auch vielfach schon geschehen ist. - In der Besprechung wurde hervorgehoben, daß über den Geschlechtsumschlag bei Ilex weitere Beobachtungen dringend erforderlich sind, und daß ein völliges Ausschalten der endozoischen Verbreitung in unseren Gegenden kaum anzunehmen ist.

Dr. W. Emeis (Kiel) berichtet über das neue Naturschutzgebiet Aarökalv.

Aarökalv ist eine etwa 2,5 ha große, aus 8 Inseln entstandene Halbinsel, die mit dem Ostrande der Insel Aarö im Kleinen Belt durch eine schmale Nehrung verbunden ist. Die Gemeinde Aarö schenkte das Gebiet unter dem Namen , $\mathrm{Na}$ turschutzgebiet von Löw" dem Landrat des Kreises Hadersleben aus Dankbarkeit für geleistete Deichbauten und für in Aussicht gestellte weiterc Schutzbauten und Verkehrsverbesserungen. Be merkenswert ist das Gebiet durch das Vorkommen des Strandflieders oder Widerstoßes (Statice bahusiensis), der hier seinen einzigen deutschen Standort hat. Sein Hauptverbreitungsgebiet liegt am Kattegatt. Daneben findet sich jetzt schon eine reiche Strandflora mit Eryngium maritimum. Echinopsilon hirsutus, Obione pedunculata, u. a. Von Kryptogamen ist die auf Lecanora glaucoma und L. sulfurea epiphytisch lebende Lecidea in tumescens besonders erwähnenswert. Dank des Schutzes hat sich im Gebiet schon eine recht reiche Vogelwelt angesiedelt.

\section{Ein neuer Beitrag zur Geschichte der Biogeographie.}

Von Prof. Dr. Walther May, Karlsruhe.

Die ältere Geschichte der Tier- und Pflanzengeographie ist bisher wenig gepflegt worden; man begnügte sich meist mit einer mechanischen Aufzählung von Namen und Tatsachen. Es ist daher freudig zu begrüßen, daß ein schwedischer Forscher, Nils von Hofsten in Upsala, neuerdings den Versuch unternommen hat, diese Lücke unseres geschichtlichen Wissens auszufüllen. Seine äußerst gründliche, auf einem umfassenden Quellenmaterial aufgebaute Arbeit „Zur älteren Geschichte des Diskontinuitätsproblems in der Biogeographie" (Zool. Annalen Bd. 7, 1916) behandelt zwar in erster Linie den Werdegang einer bestimmten biogeographischen Frage, ist aber so umfassend angelegt und beleuchtet ihren Gegenstand in so vielseitiger Weise, daß sie einen guten Einblick in die geschichtliche Entwicklung der chorologischen Ideen überhaupt gewährt.

Eine Tier- und Pflanzengeographie konnte erst aufkommen, nachdem einerseits die faunistischen und floristischen Kenntnisse eine gewisse Höho erreicht hatten, andererseits ein Bedürfnis zur $\mathrm{Er}$ klärung der Verbreitung sich geltend machte. Im Altertum fehlten diese Voraussetzungen fast ganz. Doch hatte man natürlich beobachtet, daß nicht alle Pflanzen und Tiere dieselbe Verbreitung haben, und aus dieser Erkenntnis erwuchsen die Ansätze zu einer Tier- und Pflanzengeographie. Aristoteles gibt Beispiele von Tieren mit beschränktem Vorkommen, ohne jedoch die Ursachen näher zu erörtern. Sein Schüler Theophrast hebt wiederholt hervor, daß gewisse Pflanzen in mehreren Ländern wachsen, andere dagegen einzelnen Ländern eigen sind, und $\mathrm{da} B$ verschiedene Gebiete verschiedene Gewächse hervorbringen. Für die Beobachtung, daß gewisse Tiere und Pflanzen in weit voneinander entfernten Gegenden leben und für die Erörterung der Ur sachen dieser diskontinuierlichen Verbreitung war die Zeit noch nicht gekommen. Auch die Herkunft der Inseltiere wurde noch nicht besprochen.

Im Mittelalter ging das antike Wissen von der Verbreitung der Pflanzen und Tiere auf die Araber über und rurde von ihnen wesentlich verhessert; bei mehreren arabischen Schriftstellern findet man richtige Angaben über Verbreitungsgrenzen und über die Produkte verschiedener Klimate. Für die spätere Entwicklung des Diskontinuitätsproblems und der ganzen Biogeographie liatten jedoch die im Altertum und von den $\Lambda$ rabern gewonnenen Kenntnisse der Pflanzenund Tierverbreitung keine direkte Bedeutung.

Von größerem Einfluß waren dagegen die Erörterungen des Altertums und Mittelalters über die Existenz von Antipoden und anderen in fernen Ländern wohnenden Menschen. Wenn man mehr als tausend Jahre, nachdem Augustinus die Existenz der Antipoden geleugnet hatte, wirklich Menschen jenseits des ,unermeBlichen Ozeans entdeckt hat, dann waren es die Ansichten über lie Antipoden, von denen man auszugehen hatte und auch tatsächlich ausging. Die ersten Erörterungen über die Herkunft der amerikanische Menschen und Tiere knüpfen an diejenigen über die hypothetischen Antipoden an.

Einen besonders nachhaltigen Einfluß übte $A \boldsymbol{u}$ oustinus aus, teils durch seine Behandlurg des Antipodenproblems, teils durch die Aufstellung und Beantwortung der Frage nach der Herkunft 
der Inseltiere. Er nahm eine selbständige Schöpfung vieler Inseltiere nach der Sintflut oder mit anderen Worten eine polytope Entstehung der Arten an und erwarb sich dadurch das Verdienst, das Diskontinuitätsproblem zuerst aufgestellt und seine Lösung versucht zu haben. Etwa 200 Jahre später stellte der irländische Mönch Pseudoaugustinus die Hypothese auf, daB die Säugetiere Irlands über eine ehemalige Landverbindung aus dem Kontinent eingewandert seien und erklärte damit zum erstenmal die diskontinuierliche Verbreitung gewisser Tiere durch die Annahme eines ehemaligen Zusammenhanges 2wischen heute getrennten Gebieten. Doch übte er keinen Einflu $\beta$ auf die Forschung aus, und erst die Entdeckung Amerikas drängte die Frage nach dem Ursprung der Inseltiere und Inselmenschen jedermann auf.

Die Herkunft der Menschen, Tiere und Pflanzen Amerikas gehört noch heute zu den wichtigsten und schwierigsten Fragen der Biogeographie. Für die Menschen des 16. Jahrhunderts und noch weit bis in das 18. hinein war sie jedoch ungleich bedeutungsvoller, weil sie als ein religiöses Problem aufgefaßt wurde. Angstlich sah man sich nach einer Antwort um, die den herkömmlichen Glauben ungestört ließ.

Schon im Anfang des 16. Jahrhunderts stellte Paracelsus eine Hypothese auf, die als die radikalste von allen je versuchten Lösungen des Problems bezeichnet werden muß; er nahm einen ,anderen Adam" an. Hiermit sprach er zum erstenmal die Ansicht vom polygenetischen Ursprung des Menschengeschlechtes aus, über die 300 Jahre später so viel Streit entstehen sollte. Er löste das Problem durch dasselbe Prinzip, mit dem Augustinus die Herkunft der Inseltiere erk]ärt hatte. Seine Hypothese wurde jedoch von allen Seiten als ketzerisch verworfen,- und man war genötigt, eine Einwanderung aus der Alten Welt anzunehmen, und zwar nach der Sintflut. Augustins Lehre wurde als mit der Heiligen Schrift unvereinbar erklärt; alle höheren Tiere der Erde mußten von den in der Arche geretteten Paaren herstammen.

Um die Mitte des 16. Jahrhunderts nahm ein spanischer Schriftsteller, Augustin de Zarate, eine Einwanderung des Menschen nach Amerika über eine verschwundene Atlantis an. Vielseitiger und tiefer in der Behandlung der Frage war der gelehrte Jesuit José de Acosta, dessen im 17. und 18. Jahrhundert sehr verbreitetes und beliebtes Werk über Amerika eine ausführliche Erörterung über den Ursprung der amerikanischen Menschenund Tierwelt enthält. Es gibt nach ihm nur drei Möglichkeiten: eine Besiedlung durch Schifffahrt, durch Schiffbrüchige oder durch Einwanderung über Land. Die beiden ersteren Möglichkeiten werden ausführlich widerlegt, und Acosta kommt zu dem Ergebnis, daB Menschen und Tiere über Land eingewandert seien. Hieraus zieht er den weiteren SchluB, daß die Neue Welt irgendwo mit der Alten zusammenhänge, oder daß wenigstens die Entfernung sehr unbedeutend sei, entweder im Norden oder im Süden. Die Hypotheseeiner Einwanderung über die Atlantis wird. zurückgewiesen. Acosta übte einen großen Einfluß auf die späteren Schriftsteller aus; seino klare Zurechtlegung des Problems hatte eine sichere Grundlage für die Diskussion geschaffen.

Gegen Ende des 17. Jahrhunderts veröffentlichte der berühmte und vielseitige Jesuit. Athanasius Kircher ein Buch, in dem der Gedanke an ehemalige Landverbindungen gleichsam. in ein System gebracht wurde. Damit erlahmte aber auch das Interesse für das Amerikaproblem, und es folgte eine nüchterne Periode, die dauerte, bis die französische Aufklärung einen allgemeinen Geschmask an wissenschaftlichen Speku-lationen geweckt hatte.

Jetzt wurden Buffon und Linné von hervorragender Bedeutung für die Entwicklung derBiogeographie. Jener lieferte den sicheren Nachweis, daß die Tiere des östlichen und des westlichen Kontinents in der Regel ganz verschieden sind, erkannte und erklärte die Zirkumpolarität der nördlichen Tiere und machte zuerst auf vikariierende Arten aufmerksam, deren Ähnlichkeit er aus einem gemeinsamen Ursprung, deren Verschiedenheit er aus der Einwirkung des Klimas erklärte. Linné begründete die Pflanzengeographie, die fast 200 Jahre später entstand als die ersten Anfänge der Tiergeographie. Mit besonderem Interesse schilderte der große schwedische Botaniker die diskontinuierliche Verbreitung der Gebirgspflanzen und gab viele Beispiele von Arten, die für die lappländischen, schweizerischen, pyrenäischen und andere Gebirge gemeinsam sind. Linné hat als erster diese Erscheinung erkannt, die später eine so große Bedeutung in der $\mathrm{Ge}$ schichte des Diskontinuitätsproblems erhalten sollte. Er dachte sich jedoch noch im Anschluß an die biblische Paradieserzählung eine Ausbreitung aller Pflanzen von einem einzigen Punkte aus. Demgegenüber behauptete der deutsche Botaniker Gmelin einen polytopen Ursprung der Pflanzen, wie denn überhaupt Linnés Wanderungshypothese wahrscheinlich wenige Botaniker seiner Zeit befriedigte. Gegen Ende des 18. Jahrhunderts versuchte Willdenow die diskontinuier. liche Verbreitung vieler Pflanzen durch die Annahme eines früheren Zusammenhanges zwischen den Verbreitungsbezirken zu erklären. -

Einen bedeutungsvollen Fortschritt auf dem Gebiete der Tiergeographie bezeichnet das in den Jahren 1778 bis 1783 erschienene dreibändige Werk des deutschen Universitätsprofessors Zimmermann über die ,Geographische Geschichte des Menschen und der allgemein verbreiteten vierfüßigen Tiere". Darin werden zahlreiche Fälle von diskontinuierlicher Verbreitung besprochen und durch die Hypothese eines ehemaligen Zusammenhanges jetzt getrennter Gebiete erklärt. Zimmermann war der erste, der 
diese Hypothese konsequent durchzuführen versuchte und wurde dadurch der Begründer der entwicklungsgeschichtlichen Tiergeographie. Auch schuf er den Begriff des Reliktendemismus, indem er hervorhob, daß ein kleines Verbreitungsgebiet der Rest eines früheren größeren sein kann.

Auf die deutsche Zoologie übte Zimmermann einen nicht unbedeutenden EinfluB aus; in Frankreich und England wurde er wenig beachtet. Nach seiner grundlegenden Arbeit wurde die Tiergeographie lange hauptsächlich durch die Entdeckung neuer Tatsachen gefördert; neue Geoichtspunkte konnten nicht geboten werden. -

Im Anfange des 19. Jahrhunderts nahm die Pflanzengeographie einen bedeutenden Aufschwung. In erster Linie kommen hier die Arbeiten Humboldts in Betracht, durch die eine exalite floristische und physikalische Pflanzengeographie geschaffen wurde. Humboldt kannte die diskontinuierliche Verbreitung von alpinen und anderen Pflanzen gut, sowie die Verwandtschaft zwischen weit getrennten Floren. Wie kann man sich, fragt er, Pflanzenwanderungen zwischen Gegenden vorstellen, die durch unendliche Gebiete mit ganz anderem Klima und durch den Ozean getrennt sind? Seine eigenen Ansichten sind sehr schwankend. Meist hält er solche Fragen für unlösbar; einmal nimmt er an, da $B$ dieselben Phanerogamen in Nordamerika und in Europa sowie in der nördlichen und südlichen Hemisphäre entstanden seien.

Die meisten Forscher am Anfang des 19. Jahrhunderts, die nach einer Erklärung der nicht aus dem Klima hervorgehenden Verbreitungsverhältnisse suchten, fanden diese in der Lehre von den Schöpfungszentren. Man nahm eine ursprünglich gegebene Verteilung der Arten und der systematischen Gruppen an. Bald dachte man nur an Ursprungsorte der einzelnen Arten, bald verknüpfte man damit die Vorstellung von Zentren, wo die schöpferische Tätigkeit sich besonders stark geltend gemacht habe und von denen also viele Arten ausgegangen seien. Viele waren der Ansicht, daB die jetzige Verbreitung durch Wanderungen von den Schöpfungszentren aus zustande gekommen sei, und gingen wenigstens stillschweigend von der Voraussetzung aus, daB jede Art nur an einer Stelle entstanden sei. Bei einigen dieser Autoren treten Ansätze zu einer geschichtlichen Betrachtungsweise der Verbreitungserscheinungen auf, indem sie Wanderungen in früheren Epochen mit anderer Verteilung von Land und Meer annahmen. Doch gab es auch Forscher, die von den Wanderungshypothesen nichts wissen wollten und zu dem Ergebnis kamen, daB jede Art in zahlrcichen Individuen und an verschiedenen Stellen entstanden sei.

Fin wichtiges Ereignis für die Biogeographie waren Lyells „Prinzipien der Geologie“, deren erste Auflage in den Jahren 1830 bis 1833 erschien. Ihr Verfasser stützte sich auf die ge- läufigen Annahmen von Schöpfungszentren und Wanderungen von diesen aus, betonte aber mit besonderem -Nachdruck, daB die jetzige Verbreitung wesentlich durch die geographischen und klimatischen Veränderungen der Erde beeinfluBt worden sei. Die innere Wahrheit der Ideen und die Konsequenz in ihrer Anwendung geben ihm jedenfalls einen wichtigen Platz in der Geschichte der Biogeographie; seine gröBte Bedeutung aber hat er durch seinen EinfluB auf Forbes, Hooker, De Candolle und Darwin.

Edward Forles veröffentlichte im Jahre 1846 eine Arbeit über die Flora und Fauna der britischen Inseln, die einen Wendepunkt in der Geschichte der Biogeographie bedeutet. Eine neue Richtung in dieser Wissenschaft konnte nur dadurch entstehen, daß man die großen allgemeinen Probleme beiseite ließ und die unmittelbare Herkunft der Tier- und Pflanzenwelt eines begrenzten Gebietes zu ergründen versuchte. Dies tat Forbes, indem er die Geschichte der gesamten Flora und Fauna seiner Heimat schrieb. Er geht von der Voraussetzung aus, daß jede Art sich von einem einzigen Ursprungszentrum aus verbreitet hat und zeigt ferner, da $B$ die britische Flora und Fauna wenigstens ganz überwiegend aus anderen Gegenden stammen muB. Er unterscheidet darin mehrere Elemente, die in verschiedenen Zeiten und auf verschiedenen Wegen eingewandert seien An der Bedeutung des Klimas für die Verbreitung zweifelt er nicht; das heutige Klima wird jedoch fast gar nicht berücksichtigt, und er sucht fast alles in der Verbreitung durch die Einwanderungsgeschichte zu erklären. Sein Gedankengang ist ganz überwiegend geologisch. Die Behandlung der Landflora und Landfauna berührt in jedem Punkte das Diskontinuitätsproblem. Forbes' Ansichten darin sind sehr präzis. Das britische Gebiet muB seine Pflanzen und Tiere vor der Isolierung, $d$. $h$. über verschwundene Landbrücken erhalten haben, das alpine Element sei jedoch durch Treibeis dorthin gelangt. Bei der Besprechung des alpinen Elementes und in noch höherem Grade der nördlichen Meerestiere nimmt er eine rein klimatische Isolierung an, ein in der Biogeographie neues, für die künftige Forschung außerordentlich fruchtbares Prinzip. Gleichzeitig prägte er unvergleichlich bestimmter als irgendein früherer Forscher den Reliktenbegriff.

In die FuBstapfen Forbes' traten die Botaniker Hooker und De Candolle. Jener faßte zunächst nur gewisse Erscheinungen ins Auge, dieser unterwarf in seiner klassischen „Botanischen Geographie" die Verbreitung der ganzen Pflanzenwelt einer tiefgehenden theoretischen Analyse. In der Geschichte des Diskontinuitätsproblems bildet dieses Werk einen der wichtigsten Marksteine. De Candolle schenkt der diskontinuierlichen Verbreitung größere Aufmerksamkeit als irgendein früherer Forscher; ja bis auf unsere Zeit hat eigentlich niemand die theoretische. Bedeutung 
dieser Tatsachen so eingehend und mit solch logischer Schärfe klargelegt. Immer und immer wieder kommt er darauf zurück, daß die heutigen geographischen und klimatischen Verhältnisse nur eine sehr sekundäre Rolle spielen. Die wichtigsten Züge in der Verbreitung beruhen auf früheren Ursachen; geographische und klimatische Veränderungen haben in hohem Grade darauf eingewirkt. -

Damit wurden die entwicklungsgeschichtlichen Ideen der vordarwinschen Zeit zum AbschluB gebracht. Ihnen stand jedoch eine zweite Auffassung gegenüber, deren Hauptvertreter Louis Agassiz war. Dieser betrachtete die Verbreitung als eine Eigenschaft jeder Art, wie Bau und Lebensfunktionen, also als den Ausdruck eines Gedankens des Schöpfers; er leugnete daher die Bedeutung der äußeren Bedingungen, von Wanderungen usw. Die jetzige Verbreitung jeder Art ist seit ihrer Schöpfung gleich gewesen. Jede Art ist nicht nur innerhalb ihres jetzigen Wohngebietes entstanden, sondern über dessen ganze Ausdehnung und in großer Anzahl, sogar in durchschnittlich derselben Anzahl von Individuen, die sie jetzt aufweist und die eines ihrer angeborenen Merkmale ist. Einen tieferen Einfluß auf die biogeographische Forschung übte Agassiz nicht aus. Doch ist Schmardas großes Werk über die Verbreitung der Tiere ein typischer Ausdruck der von dem amerikanischen Zoologen abhängigen Richtung. In seinem Mangel an entwicklungsgeschichtlichen und sonstigen allgemeinen Gesichtspunkten zeigt es klar, wie wenig die Tiergeographie eigentlich seit Zimmermanns Tagen fortgeschritten war; Forbes' Ideen wirkten dort viel weniger als in der Botanik.

Sowohl die Forbes-De Candollesche als auch die Agassizsche Richtung, zwischen denen der Botaniker Grisebach einen vermittelnden Standpunkt einnahm, setzten als tiefste Ursache der Verbreitungserscheinungen eine ursprüngliche, nach übersinnlichen oder wenigstens unerklärlíchen Gesetzen erfolgte Verteilung der Organismen voraus. Forbes und $D e$ Candolle nahmen spätere Veränderungen an und sahen also in der jetzigen Verbreitung das Ergebnis einer Entwicklung; Agassiz betrachtete alle Verbreitungserscheinungen als von Anfang an gegeben. Seine Anschauung hatte den Vorzug der Folgerichtigkeit, sie beantwortete ein für allemal alle Fragen. Dagegen enthielt die entwicklungsgeschichtliche Auffassung jener Zeit einen inneren Widerspruch: sie erklärte gewisse Einzelheiten in der Verbreitung als Resultate einer Entwicklung, schlug dann plötzlich um und führte die wesentlichsten Züge in der Verteilung der Organismen auf einen ursprünglichen, von übersinnlichen Prinzipien bedingten Zustand zurück. Sie konnte nicht klarlegen, warum nicht ebensogut alles der Ausdruck dieser geheimnisvollen Gesetze sei. Da kam die Deszendenztheorie auf. Die Agassizsche Lehre war auf einmal überwunden, der Widerspruch ge- löst, und die entwicklungsgeschichtliche Auffassung der Verbreitung erfuhr eine folgerichtige Ausgestaltung und erhielt einen tieferen Sinn. Agassiz überlebte seine Lehre; bei seinem l'ode hatte die genetische Biogeographie vollständig gesiegt. -

Der vorstehende Auszug aus der prächtigen Arbeit $v$. Hofstens kann nur einen annähernden Begriff von der Reichhaltigkeit ihres Inhalts geben. Ganze Abschnitte, wie die über dio Antipodenfrage, die Beobachtungen über Inseltiere im 17. und 18. Jahrhundert, die Salonwissenschaft am Ende des 18. Jahrhunderts, den anthropologischen Polygenismus, die miozäne Atlantis, Wallace, Darwin, die Jahre nach Darwin und das moderne Problem der polytopen Artentstehung mußte ich hier ganz übergehen. Bezüglich ihrer und vieler interessanter Einzelheiten sei auf die grundlegende Studie selbst verwiesen.

\section{Zur Stammesgeschichte der Halbaffen und Menschenaffen.}

Kein Gebiet der Stammesgeschichte erweckt allgemeineres Interesse als die Entwicklung der Halbatifen und Affen, als der Lebewesen, die uns Menschen olino Zweifel von allen am nüchstea stehen und deren Entwicklungsgeschichte zu eincm großen Teile auch unsere Geschichte ist. Dabei haben freilich die Ansichten im Laufe der Zeit stark gewechselt und besonders uber die Beziehungen der Halbuffen zu den Affen und ihre Gliederung sind z. T. weit auseinandergehende Ansichten geïuBert worden. So ist auch hier unser Wissen noch immer im Flusse und jeder gründlich bearbeitete Beitrag wertvoll. W. $K$. Gregory, der sich schon als paliiontologischer Bearbeiter der Süugetiersystematik einen Namen gemacht hat, hat sich nun in neuerer Zeit eingehender mit der Stammesgeschichte der Halbaffen und Menschenaffen beschïftirt und ist dabei zu manchen neuen wertvollen Resultaten gelangt, die es verdienen, auch weiteren Kreisen zugänglich gemacht $z u$ werden.

Was zunächst die Halbaffen anlangt1), so kommt auch Gregory zu dem Ergebnis, daß der malaiische Gespenstmaki (Tarsius) unter den lebenden Tieren dieser Unterordnung eine besondere Stellung einnimmt. Ihm sind nïchstverwandt die Anaptomorphiden, die im Eozilin und Unteroligozïn in zahlreichen Gattungen und Arten in Norlamerika lebten. AuBerdem stehen ihnen aber auch nach Gregory die Microchörinen mit den Gattungen Microchoerus und Necrolemur aus dem Unteroligoziin Europas nahe, von denen besonders der letztere in seinem Schïdelbau in vieler Hinsicht starke Anklïnge an die Koboldmakis aufweist. Die hierher gehörigen Halbaffen waren also sicher im Alttertiär weit über die nördliche Halbkugel verbreitet und sind erst später auf ihr heutiges kleines Wohngebiet beschrïnkt worden.

Neben dieser Sektion der Gespenstmakis stehen nun' zunächst die Makis (Lemuriformen), die leute

1) W. $K$. Gregory, On the Relationship of the Eocene Lemur Notharctus to the Adupiduc and to other Primates. - On the Classification and Pliylogeny of the Lemuroidea. Bull. Geol. Soc. Am. XX̃VI, 1915, p. 419-446. 